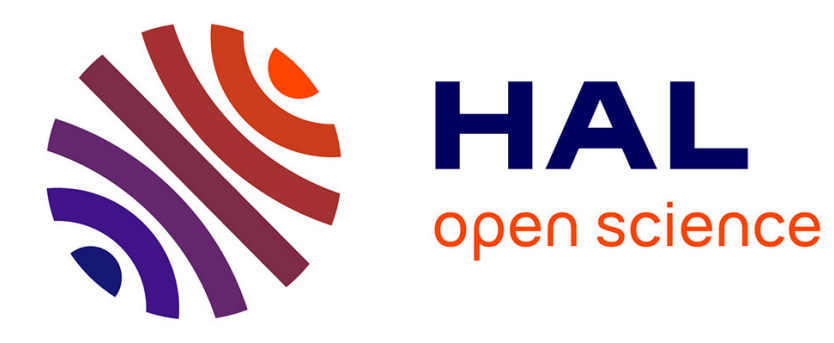

\title{
Visualisation des champs ultrasonores par interférométrie hétérodyne
}

\author{
O. Casula, D. Royer
}

\section{To cite this version:}

O. Casula, D. Royer. Visualisation des champs ultrasonores par interférométrie hétérodyne. Journal de Physique IV Proceedings, 1994, 04 (C5), pp.C5-1217-C5-1220. 10.1051/jp4:19945269 • jpa-00252959

\section{HAL Id: jpa-00252959 https://hal.science/jpa-00252959}

Submitted on 1 Jan 1994

HAL is a multi-disciplinary open access archive for the deposit and dissemination of scientific research documents, whether they are published or not. The documents may come from teaching and research institutions in France or abroad, or from public or private research centers.
L'archive ouverte pluridisciplinaire HAL, est destinée au dépôt et à la diffusion de documents scientifiques de niveau recherche, publiés ou non, émanant des établissements d'enseignement et de recherche français ou étrangers, des laboratoires publics ou privés. 


\title{
Visualisation des champs ultrasonores par interférométrie hétérodyne
}

\author{
O. CASULA et D. ROYER
}

Laboratoire Ondes et Acoustique de l'Université Paris 7, URA 1503 du CNRS, ESPCI, 10 rue Vauquelin, 75231 Paris cedex 05, France

\begin{abstract}
A high resolution optical system has been designed and developed for imaging pulsed ultrasonic fields transmitted in water by piezoelectric transducers. The particle displacement was measured by a compact heterodyne interferometer whose probe beam is reflected by a thin membrane immersed in front of the transducer. Experiments carried out on focused or planar transducers demonstrate the capability of the system to map acoustic fields both in time and spatial domains. The spatial resolution and the dynamic range reached $20 \mu \mathrm{m}$ and $45 \mathrm{~dB}$ and the bandwidth can be enlarged up to $40 \mathrm{MHz}$. Results are presented in various cross sectional views (B-scans, C-scans and 3D profiles).
\end{abstract}

\section{1- INTRODUCTION}

La conception et la calibration des capteurs ultrasonores utilisés pour les applications industrielles (CND) et médicales nécessitent la mesure, avec une grande résolution spatiale et temporelle, des champs acoustiques impulsionnels rayonnés par les transducteurs piézoélectriques. Les méthodes "classiques" utilisant un réflecteur en forme de bille ou un hydrophone miniature sont limitées aussi bien du point de vue de leur bande passante (10 MHz) que des dimensions latérales de la sonde $(0,3 \mathrm{~mm})$.

Etant donné leurs avantages potentiels (mesure absolue non perturbatrice, résolutions spatiale et temporelle pouvant atteindre respectivement $10 \mu \mathrm{m}$ et $10 \mathrm{~ns}$ ) les méthodes optiques sont a priori séduisantes $[1,2]$.

\section{2- DISPOSITIF EXPERIMENTAL}

Le dispositif expérimental (figure 1) comprend une fine membrane réfléchissante de mylar ( $\varnothing 40-100$ $\mathrm{mm}$, épaisseur 3-15 $\mu \mathrm{m}$ ) immergée dans l'eau en face du transducteur et une sonde optique du type MachZehnder hétérodyne [3]. Le faisceau sonde, dont la fréquence est décalée par un modulateur acoustooptique est focalisé sur la membrane; le mouvement de celle-ci, induit par le passage de l'onde de pression, module la phase du faisceau réfléchi. Le battement de ce faisceau, sur la photodiode, avec le faisceau de référence et une démodulation cohérente du photocourant par une électronique à large bande passante (30 $\mathrm{kHz}-40 \mathrm{MHz}$ ) fournit un signal électrique proportionnel au déplacement mécanique. Pour relever le champ acoustique, le transducteur est déplacé parallèlement à la membrane avec un pas de $10 \mu \mathrm{m}$ sur une surface pouvant atteindre $70 \times 70 \mathrm{~mm}$. Après numérisation, les signaux sont traités et enregistrés par un PC-486. Le champ acoustique peut être ensuite présenté suivant différents modes (B-scan, C-scan, vue 3D) à l'aide d'un logiciel approprié.

La figure 2 montre la distribution des vitesses, mesurée sur une membrane d'épaisseur $3 \mu \mathrm{m}$ placée dans le plan focal d'un transducteur (Panametrics V324) de fréquence centrale $25 \mathrm{MHz}$, d'ouverture 6,35 $\mathrm{mm}$ et de distance focale $18 \mathrm{~mm}$. La surface $(1,5 \times 1,5 \mathrm{~mm})$ est explorée par pas de $20 \mu \mathrm{m}$. Le diamètre mesuré de la tache focale $(0,28 \mathrm{~mm}$ à $-6 \mathrm{~dB})$ correspond bien à la valeur prévue $(0,24 \mathrm{~mm})$. 

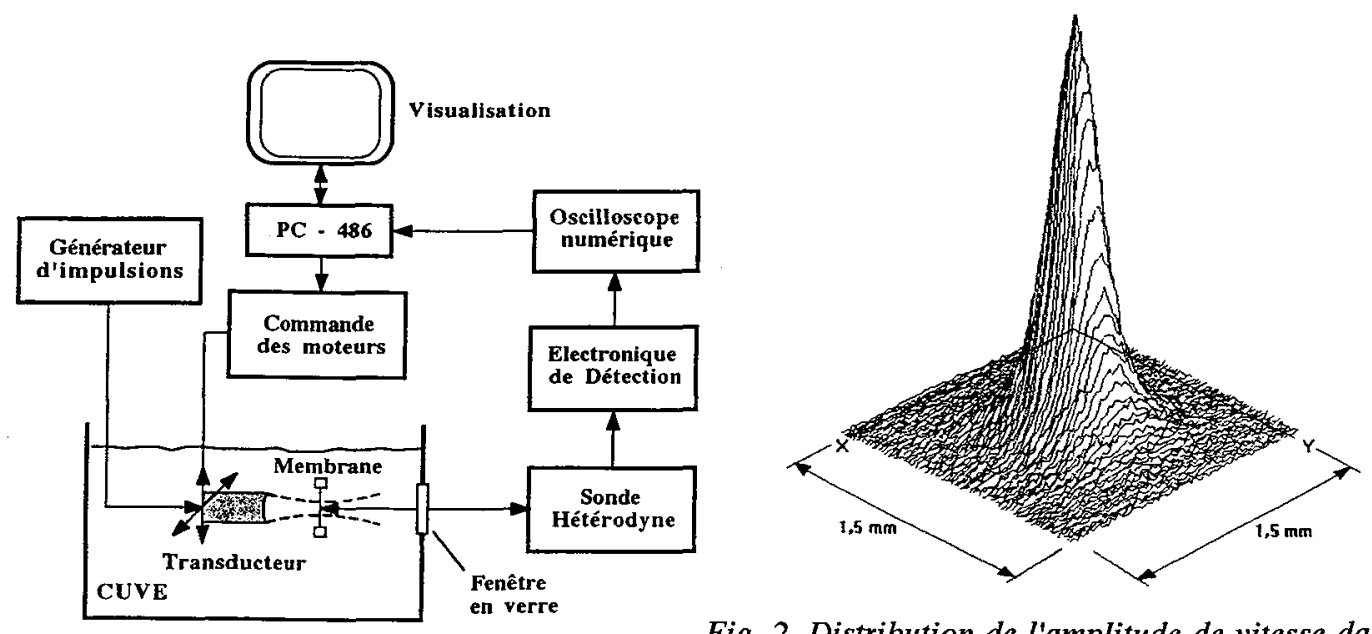

Fig. 2. Distribution de l'amplitude de vitesse dans le plan focal d'un tranducteur de fréquence $25 \mathrm{MHz}$ et Fig. 1. Dispositif expérimental. de distance focale $18 \mathrm{~mm}$. Le pas de mesure est $20 \mu \mathrm{m}$.

La mesure absolue des déplacements mécaniques par la sonde hétérodyne dépend à la fois de la réponse de la membrane et de l'interaction acousto-optique. Examinons ces deux effets.

\section{3- INFLUENCE DE L'EPAISSEUR DE LA MEMBRANE}

La réponse spatio-temporelle de la membrane à une distribution de pression rectangulaire ou parabolique a été modélisée [4]. Le résultat de la simulation est qu'une membrane de mylar d'épaisseur $6 \mu \mathrm{m}$ suit fidèlement les déplacements acoustiques du fluide jusqu'à des fréquences de $20 \mathrm{MHz}$. Expérimentalement, nous avons relevé le déplacement au foyer du transducteur de fréquence centrale $25 \mathrm{MHz}$ sur trois membranes d'épaisseurs différentes $(3,6$ et $15 \mu \mathrm{m})$. La figure 3a montre que les réponses des membranes de 3 et $6 \mu \mathrm{m}$ sont très proches, alors que l'amplitude crête-crête du déplacement mesuré sur la membrane de $15 \mu \mathrm{m}$ est diminuée de $20 \%$. Les spectres de la vitesse (figure $3 \mathrm{~b}$ ) mettent en évidence la bande passante du système $(40 \mathrm{MHz})$ et l'atténuation apportée par la membrane de $15 \mu \mathrm{m}$ au delà de $10 \mathrm{MHz}$.

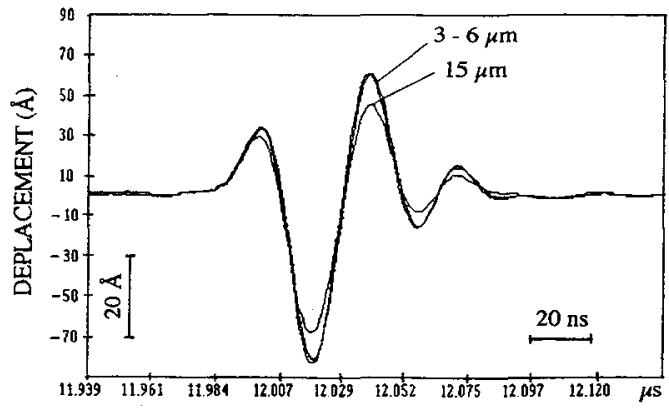

(a)

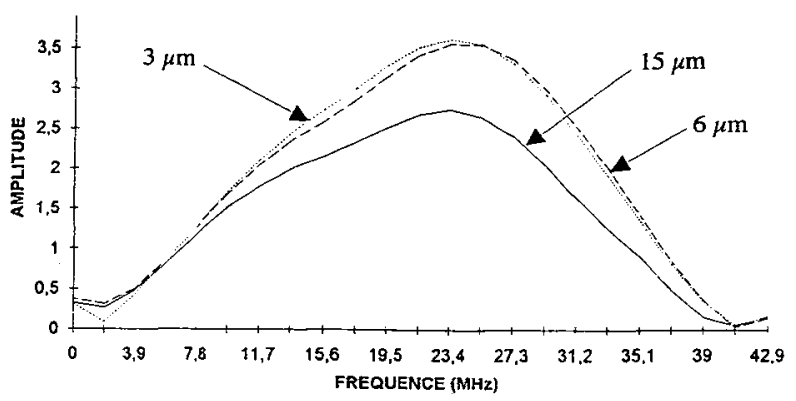

(b)

Fig. 3. a) Déplacement mécanique mesuré au foyer d'un transducteur de fréquence centrale $25 \mathrm{MHz}$ sur trois membranes d'épaisseurs différentes $(3,6$ et $15 \mu \mathrm{m})$. b) Spectres.

\section{4 - EFFET DE L'INTERACTION ACOUSTO-OPTIQUE}

L'onde ultrasonore qui a traversé la membrane modifie, par l'intermédiaire du coefficient piézo-optique $\mu$, l'indice optique $\mathrm{n}$ du milieu où se propage le faisceau lumineux sonde :

$$
\mathrm{n}=\mathrm{n}_{0}+\mu \mathrm{p}(\overrightarrow{\mathrm{r}}, \mathrm{t})
$$

La sonde est sensible au déphasage $\Delta \Phi$ subi par le faisceau lumineux lors de son parcours entre la face 
interne de la fenêtre en verre située à $z_{0}+L$, supposée immobile $\left[u_{z}\left(z_{0}+L\right)=0\right]$ et la membrane située en $z_{0}$ dont le déplacement normal est $u_{0}(t)=u_{z}\left(z_{0}, t\right)$

$$
\Delta \phi=-\frac{4 \pi}{\Lambda_{0}}\left[\int_{z_{0}+u_{0}(t)}^{z_{0}+L} n(\vec{r}, t) d z-n_{0} L\right]=\frac{4 \pi}{\Lambda_{0}} n_{0} u_{0}(t)-\frac{4 \pi}{\Lambda_{0}} \mu \int_{z_{0}}^{z_{0}+L} p(\vec{r}, t) d z
$$

La pression acoustique et la composante $\mathrm{u}_{\mathrm{z}}$ du déplacement s'expriment à l'aide de la réponse impulsionnelle de diffraction $h(\vec{r}, t)$. Dans le cas d'un piston plan circulaire de rayon a et de déplacement normal à surface $u_{n}(t)$, il vient

$$
p(z, t)=\rho_{0} c\left[v_{n}\left(t-\frac{z}{c}\right)-v_{n}\left(t-\frac{\sqrt{z^{2}+a^{2}}}{c}\right)\right] \quad \text { et } \quad u_{z}(z, t)=u_{n}\left(t-\frac{z}{c}\right)-\frac{z}{\sqrt{z^{2}+a^{2}}} u_{n}\left(t-\frac{\sqrt{z^{2}+a^{2}}}{c}\right)
$$

En dérivant $\mathrm{u}_{\mathrm{z}}$ par rapport à $\mathrm{z}$, la pression acoustique se met sous la forme

$$
\mathrm{p}(\mathrm{z}, \mathrm{t})=-\rho_{0} \mathrm{c}^{2} \frac{\partial \mathrm{u}_{\mathrm{z}}}{\partial \mathrm{z}}-\rho_{0} \mathrm{c}^{2} \frac{\mathrm{a}^{2}}{\left(\mathrm{z}^{2}+\mathrm{a}^{2}\right)^{3 / 2}} \mathrm{u}_{\mathrm{n}}\left(\mathrm{t}-\frac{\sqrt{\mathrm{z}^{2}+\mathrm{a}^{2}}}{\mathrm{c}}\right)-\rho_{0} \mathrm{c} \frac{\mathrm{a}^{2}}{\mathrm{z}^{2}+\mathrm{a}^{2}} \mathrm{v}_{\mathrm{n}}\left(\mathrm{t}-\frac{\sqrt{\mathrm{z}^{2}+\mathrm{a}^{2}}}{\mathrm{c}}\right)
$$

Le déphasage est donné par la relation 2 qui s'écrit, après substitution et compte tenu des conditions aux limites en $\mathrm{z}_{0}$ et $\mathrm{z}_{0}+\mathrm{L}$ :

$\Delta \phi=\frac{4 \pi}{\Lambda_{0}}\left[\left(n_{0}-\mu \rho_{0} c^{2}\right) u_{0}(t)+\int_{z_{0}}^{z_{0}+L} \frac{\mu \rho_{0} c^{2} a^{2}}{\left(z^{2}+a^{2}\right)^{3 / 2}} u_{n}\left(t-\frac{\sqrt{z^{2}+a^{2}}}{c}\right) d z+\int_{z_{0}}^{z_{0}+L} \frac{\mu \rho_{0} c a^{2}}{z^{2}+a^{2}} v_{n}\left(t-\frac{\sqrt{z^{2}+a^{2}}}{c}\right) d z\right](5)$

Lorsque la membrane est très proche du transducteur et la fenêtre très loin, les bornes d'intégration peuvent être remplacées par 0 et l'infini. L'expression 5 se met sous la forme d'un produit de convolution:

$$
\Delta \phi(t)=\frac{4 \pi}{\Lambda_{0}}\left[n_{e} u_{n}(t)+\mu \rho_{0} c^{2}\left(\frac{a^{2}}{c t^{2}} \frac{1}{\sqrt{c^{2} t^{2}-a^{2}}}\right) \otimes u_{n}(t)+\mu \rho_{0} c^{2}\left(\frac{a^{2}}{c t} \frac{1}{\sqrt{c^{2} t^{2}-a^{2}}}\right) \otimes v_{n}(t)\right],
$$

avec la condition $t>a / c$ pour les deux derniers termes. L'effet de l'interaction acousto-optique sur l'onde plane (premier terme) se traduit par un simple changement d'indice [5]: l'indice de l'eau $\left(\mathrm{n}_{0}=1,33\right)$ est remplacé par un indice effectif $n_{e}=n_{0}-\mu \rho_{0} c^{2}$ dont les valeurs théorique $(1,033)$ et expérimentale $(1,015)$ sont très proches de l'indice de l'air [4]. La simulation de la figure $4 \mathrm{a}$, confirmée par l'expérience (figure 4 b) montre, d'une part que les contributions de l'onde de bord (2ème et 3ème termes de l'équation 5) et de l'onde plane dans le déphasage $\Delta \phi(t)$ sont de même signe alors que pour le déplacement elles sont de signes opposés (formule $3 \mathrm{~b}$ ) et, d'autre part que l'interaction acousto-optique renforce considérablement l'importance de l'onde de bord. Lorsque la distance $\mathrm{z}_{0}$ de la membrane à la surface du transducteur est petite devant le rayon a du disque (ici $\mathrm{z}_{0}=0,7 \mathrm{~mm}$ et $\mathrm{a}=6,5 \mathrm{~mm}$ ), la quasi-totalité du signal d'onde de bord provient de l'interaction acousto-optique: dans le déphasage $\Delta \phi$, mesuré par la sonde, la contribution du déplacement normal de la membrane est négligeable.

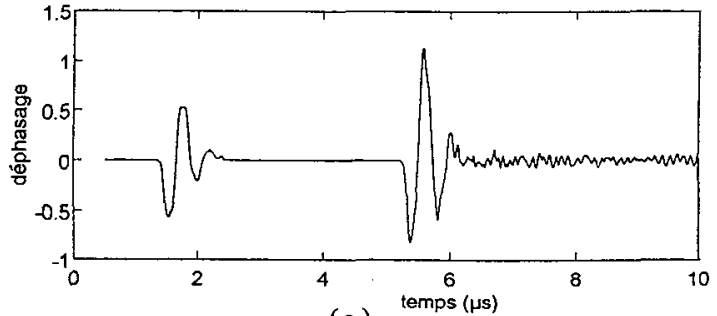

(a)

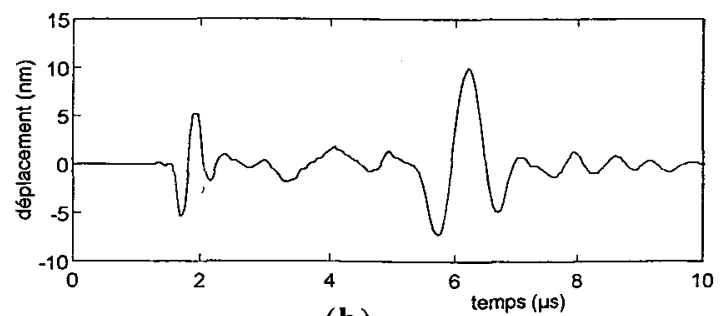

(b)

Fig. 4. Transducteur plan $\left(f=2,25 \mathrm{MHz}, a=6,5 \mathrm{~mm}, z_{0}=0,7 \mathrm{~mm}\right)$. a) Effet de l'interaction acoustooptique sur le déphasage optique (simulation). b) Relevé effectué avec la sonde hétérodyne. 


\section{5- CARACTERISATION DE TRANSDUCTEURS}

Le dispositif interférométrique décrit précédemment peut être utilisé pour caractériser les transducteurs en plaçant la membrane très près de la surface. Le profil des déplacements mécaniques de la figure $5 \mathrm{a}$, mesuré sur une membrane de $15 \mu \mathrm{m}$ située à $0,5 \mathrm{~mm}$ de la surface d'un transducteur plan (Panametrics $\mathrm{V} 114, \varnothing 22 \mathrm{~mm}, \mathrm{f}=1 \mathrm{MHz}$ ), montre que celui fonctionne bien comme un piston. Le déplacement, unipolaire, est uniforme et très amorti (figure $5 b$ ), excepté en regard d'une petite zone $(\varnothing \approx 2 \mathrm{~mm}$ ) où il est plus important et où il oscille plus longtemps (figure $5 \mathrm{c}$ ). Ce défaut est vraisemblablement dù à une bulle d'air existant entre le milieu amortisseur et la céramique piézoélectrique qui réduit localement l'amortissement. Il apparaît aussi une bande (horizontale) caractérisée par un déplacement légèrement plus faible que celui du mode piston. Cette réduction correspond à l'effet de masse produit par la bande conductrice pressée sur l'électrode externe de la céramique pour réaliser le contact électrique.

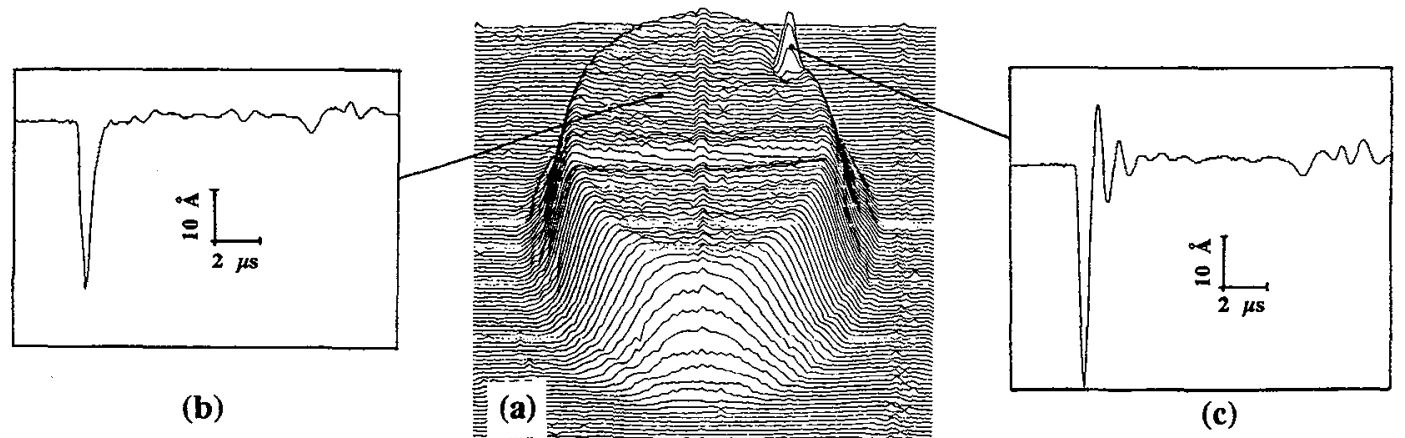

Fig. 5. Profil du déplacement mécanique mesuré à $0,5 \mathrm{~mm}$ de la surface d'un transducteur plan montrant un défaut ponctuel (a). Déplacement en face d'un point quelconque de la surface active (b). Déplacement en regard du défaut (c).

\section{6- CONCLUSION}

Les champs acoustiques rayonnés dans l'eau par des transducteurs piezoélectriques ont été relevés par un dispositif d'imagerie optique comprenant une membrane et un interféromètre hétérodyne. Les caractéristiques du système sont les suivantes: bande passante 0,1 à $40 \mathrm{MHz}$, résolution spatiale $20 \mu \mathrm{m}$, sensibilité $0,1 \AA$, surface balayée $70 \times 70 \mathrm{~mm}$. L'influence de l'épaisseur de la membrane sur la réponse spatio-temporelle du système a été modélisée et vérifiée expérimentalement. L'effet de l'interaction acoustooptique dans l'eau, tout au long du faisceau lumineux de la sonde hétérodyne, a été pris en compte dans le cas d'un transducteur plan vibrant en mode piston. L'analyse montre que l'onde plane et l'onde de bord ne sont pas affectées de la même manière par cette interaction.

Des cartographies du champ acoustique rayonné par des transducteurs plans ou focalisés ont été dressées. L'examen de plusieurs transducteurs du type de ceux utilisés en CND a mis en évidence dans certain cas des anomalies plus ou moins importantes dans le champ ultrasonore rayonné.

\section{REFERENCES}

1 - Higgins, F. P., Norton, S.J., and Linzer, M., "Optical interferometric visualization and computerized reconstruction of ultrasonic fields", J. Acoust. Soc. Am., 68, p.1169 (1980).

2 - Nagaï, S. "Acoustic power measurement using an optical heterodyne method", Ultrasonics p.77 (1985)

3 - Royer, D. and Dieulesaint, E., "Optical probing of the mechanical impulse response of a transducer", Appl. Phys. Lett., 49, 1056 (1986).

4 - Royer, D, Dubois, N. and Benoist, Ph.," Optical probing of acoustic fields - Application to the ultrasonic testing of steam generator tubes" IEEE Ultrason. Symp. Proc. p. 805 (1992).

5 - Bacon, D. R., "Primary calibration of ultrasonic hydrophones using optical interferometry" IEEE Trans. Ultrason., Ferroelect. and Freq. Control, 35, 152 (1988). 